\title{
Lycopene inhibits mitogen-activated lymphocyte activity by modulating T helper type 1 and type 17 activities
}

\author{
C.G. Alvarez Campano, F. Ward, S. Moir and F. Thies \\ The Rowett Institute, University of Aberdeen, Aberdeen AB25 2ZD
}

Atherosclerotic cardiovascular disease is a prominent cause of morbidity and mortality worldwide and a national clinical priority in Scotland ${ }^{(1)}$. Considering the central role played by inflammation in atherogenesis, identifying potential means to inhibit this process is paramount for the treatment and prevention of cardiovascular disease. T helper lymphocytes are central to this process, and previous work showed that lycopene can inhibit lymphocyte proliferation ${ }^{(2)}$. However, the effects of lycopene on T lymphocyte subsets remain to be elucidated, particularly with regards to regulatory $\mathrm{T}$ cells, which play a central role in the modulation of immune responses including inflammation ${ }^{(3)}$. Therefore, this project aimed to assess the effect of lycopene on $\mathrm{T}$ helper and $\mathrm{T}$ regulatory cells activity.

All experiments were conducted in-vitro using peripheral blood mononuclear cells isolated from healthy volunteers. The cells were incubated for 5 and 10 days with or without anti-CD3, in the presence of lycopene-enriched $(5 \mu \mathrm{g} / \mathrm{mL})$ or lycopene-free liposomes. Furthermore, the experiments were conducted in the presence of interleukin 2 to promote the expansion of the $\mathrm{T}$ regulatory cells. $\mathrm{T}$ regulatory population, as well as T helper response (types 1,2 and 17) related activities were assessed by using specific cell surface markers visualised by flow cytometry and by measuring particular cytokines (interleukins 4, 10, 17 and interferon gamma). Additionally, the expression of kynurenine 3-monooxygenase was measured as a marker of T regulatory cells activity.

Lycopene significantly inhibited $\mathrm{T}$ helper 1 and $\mathrm{T}$ helper 17 responses both after 5 and 10 days culture, evidenced by a decrease in the secretion of IFN- $\gamma$ and IL-17, respectively. Additionally, lycopene tended to increase the Foxp $3^{+}$T regulatory cell population after 5 days of incubation and tended also to increase kynurenine 3-monooxygenase, a marker of regulatory $\mathrm{T}$ cell activity, at both time points.

Effect of lycopene on IFN- $\gamma$ and IL-17 concentrations (Table) and on Foxp3 ${ }^{+}$Treg cells (Figure)

\begin{tabular}{|l|c|l|}
\hline & $\begin{array}{c}\mathrm{IFN} \gamma \\
(\mathrm{pg} / \mathrm{mL})\end{array}$ & $\mathrm{IL}-17(\mathrm{pg} / \mathrm{mL})$ \\
\hline Control & $\begin{array}{c}34133 \\
(5440)\end{array}$ & $1292^{*}(328)$ \\
\hline Lycopene & $\begin{array}{l}24476 \\
(5114)\end{array}$ & $737 *(258)$ \\
\hline
\end{tabular}

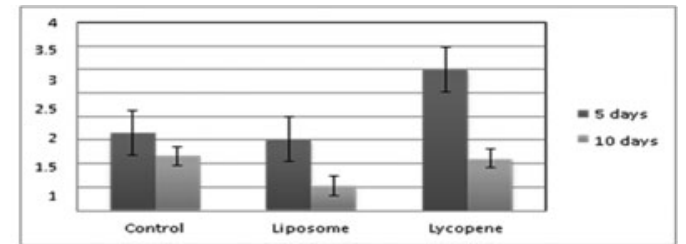

Values are mean (SD), $n=6$, after 10 days culture with antiCD3. Differences between the groups were assessed by univariate analysis.* Significantly different from control $(p<0.05$, Tukey's test). In the figure, data is presented as a stimulation index (ratio of the anti-CD3 activated cells by the non-activated control) + SEM for both 5 and 10 days incubation.

These results show that lycopene inhibits $\mathrm{T}$ helper type 1 and type 17 responses, possibly by modulating regulatory $\mathrm{T}$ cell activity. These results highlight novel potential mechanisms by which lycopene could exert cardiovascular protection.

\section{Acknowledgements}

Mexican Council for Science and Technology (CONACYT), Institute for Innovation and Technology Transfer $\left(\mathrm{I}^{2} \mathrm{~T}^{2}\right)$ and the Mexican Ministry of Education (SEP).

1. ISD. Information Services Division - NHS Scotland (2016) Scottish Heart Disease Statistics. Available at: www.isdscotland.org/Health-Topics/ Heart-Disease/Publications/2016-01-26/2016-01-26-Heart-Disease-Summary.pdf (Accessed: 15 January 2017).

2. Mills LM, Wilson H and Thies F (2012) Molecular Nutrition \& Food Research 56(7), 1034-42.

3. Wolf D, Zirlik A and Ley K (2015) Cellular and Molecular Life Sciences 72(20), 3853-69. 